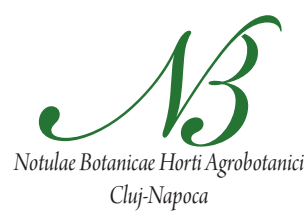

\title{
Identification, Characterization, and Expression Analysis of the TaNF-YB3 Gene from Triticum aestivum
}

\author{
Xiuchun DONG ${ }^{1}$, Zhiyuan $\mathrm{CHENG}^{1}$, Shuhan $\mathrm{CHENG}^{2 *}$, Feng \\ $\mathrm{XU}^{3}$, Yanrong $\mathrm{AN}^{1}$, Xizuo $\mathrm{BIAN}^{1}$, Ting $\mathrm{CHEN}^{1}$ \\ ${ }^{1}$ Shandong Agricultural University, College of Life Sciences, Taian, Shandong 271018, China; \\ ${ }^{2}$ Shandong Agricultural University, College of Information Science and Engineering, Taian, \\ Shandong 271018, China; shcheng@sdau.edu.cn (" corresponding author) \\ ${ }^{3}$ Yangtze University, College of Horticulture and Gardening, Jingzhou 434025, China
}

\begin{abstract}
A full-length cDNA encoding a nuclear factor-YB (NF-YB)/HAP3/CCAAT binding factor-A (CBF-A) subunit of a CCAAT-box binding complex, designated as TaNF-YB3 was isolated from Triticum aestivum. Sequence analysis indicated that the full-length cDNA was 809 bp long, including an open reading frame (ORF) of $597 \mathrm{bp}$, which encoded a deduced polypeptide of 199 amino acids and is located in chromosome 3D. The deduced protein contained conserved structural domains and showed high identity to other plant NF-YBs. TaNF-YB3 was expressed in various organs, especially in the leaves and stamens; it was also regulated by salt, mannitol, abscisic acid, wounding, and cold. Moreover, TaNF-YB3 was down-regulated by short days and vernalization, and sensitive to the transfer of day length. It was mainly induced by light and exhibited a similar diurnal rhythmic expression pattern with the CCT-domain family gene VRN2 (TaZCCT1 and TaZCCT2), but not with CO (WCO1 and TaHd1). Overall, the results suggested that TaNF-YB3, aside from having a role in regulating day length and vernalization responses, might integrate signals from other environmental stresses to perform its functions in winter wheat adaptability and development.
\end{abstract}

Keywords: expression analysis, TaNF-YB3, Triticum aestivum, cloning

\section{Introduction}

The CCAAT-box is one of the most common elements in eukaryotic promoters. It is present in $30 \%$ of all eukaryotic genes and is typically found between 60 and 100 bp upstream of the transcription start site (Bucher, 1990; Dorn et al., 1987; Edwards et al., 1998; Maity and de Crombrugghe, 1998; Mantovani, 1998; Mantovani, 1999). The nuclear factor-Y (NF-Y) complex, isolated as a CCAAT-binding protein complex, is an evolutionarily conserved transcription factor that occurs in a wide range of organisms, from yeast to humans. It consists of three distinct subunits, namely NF-YA (also called HAP2 or CBF-B), NF-YB (HAP3/CBF-A), and NF-YC (HAP5/ CBF-C) (Romier et al., 2003). Each subunit is required for DNA binding (Mantovani, 1999). A fourth subunit, HAP4 is present in yeast; it does not bind to DNA but is required for complex formation (Forsburg and Guarente, 1989; McNabb et al., 1997).

In animals and yeast, a single gene of each NF-Y subunit exists, whereas plants have multiple genes of each subunit. In the dicotyledonous plant Arabidopsis, there are 10,10 , and 9 genes encoding the NF-YA (HAP2/CBF-B), NF-YB (HAP3/CBF-A), and NF-YC (HAP5/CBF-C) subunits, respectively (Edwards et al., 1998; Gusmaroli et al., 2001, 2002). In monocotyledonous plants, 10 genes for NF-YA, 1 lgenes for NF-YB, and 13 genes for NF-YC have been recognized in rice (Miyoshi et al., 2003; Thiruvengadam et al., 2008). Furthermore, 10 putative NF-YA, 11 putative NF-YB genes, and 14 putative NF-YC genes have been identified in Triticum aestivum (Stephenson et al., 2007).

With regard to plant, several single plant NF-Y subunits, known to have functions in embryogenesis, drought resistance, abscissic acid ( $\mathrm{ABA}$ ) signaling, nitrogen-fixing nodule development, and flowering time (Combier et al., 2006; Kwong et al., 2003; Li et al., 2008; Lotan et al., 1998; Nelson et al., 2007; Warpeha et al., 2007) have been studied in detail in recent years. The first was isolated from maize as a HAP3 homolog (Li et al., 1992). Another single ZmNF-YB subunit gene in maize confers drought tolerance, and its transgenic maize overexpression in plants improves corn yields under water-limited conditions ( $\mathrm{Nel}$ son et al., 2007). Li et al. (2008) reported that the Arabidopsis NF-YA5 transcription factor is transcriptionally and post-transcriptionally regulated to promote drought resistance. Furthermore, LEC1 and LEC1-LIKE (LIL) of the Arabidopsis HAP3 subunits play important roles in 
embryogenesis (Lee et al., 2003; Lotan et al., 1998; Kwong et al., 2003). In carrot, $C-L E C 1$, a functional orthologue of $L E C 1$, was suggested to play a similar role in embryogenesis (Yazawa et al., 2004). In other species, MtHAP2-1 plays a key role in symbiotic nodule development regulated by microRNA169 in Medicago truncatula (Combier et al., 2006). Three OsHAP3 subunits (OsHAP3A, OsHAP3B, and $\mathrm{OsHAP3C}$ ) of rice function during chloroplast development (Miyoshi et al., 2003).

Most recently, several publications reported that NF-Y transcription factors are intimately involved in photoperiod-dependent flowering. Ben-Naim et al. (2006) reported that overexpression of tomato (Solanum lycopersicum) HAPSa in Arabidopsis caused early flowering. In contrast, overexpression of Arabidopsis HAP3a and HAPSa delayed flowering (Wenkel et al., 2006). The authors showed evidence that the CCT-domain of the CONSTANS (CO), CONSTANS-LIKE, and TOC1 proteins replace AtHAP2 subunits to form a trimeric CO/AtHAP3/ AtHAP5 complex that regulates gene expression. The NF-YB2 subunit (HAP3b) has been shown to promote flowering through the activation of the floral integrators FLOWERING LOCUS T $(F T)$ and SUPPRESSOR OF OVEREXPRESSION OF CO 1 (SOC1) (Cai et al., 2007; Chen et al., 2007). These effects were apparent only under long-day conditions, and suggest that $\mathrm{CO}$ interacts with DNA through an NF-Y platform both in vitro and in vivo. In Arabidopsis, NF-YB2 and NF-YB3 have been shown to play additional roles in the promotion of flowering by inductive long-day photoperiods (Kumimoto et al., 2008). They provided a novel demonstration that plant NF-YB subunits are capable of directly binding to a CCAAT-box containing a region of the FT promoter as part of an NF-Y trimer in combination with the yeast HAP2 and HAP5 subunits. Kumimoto et al. (2010) recently reported that three Arabidopsis proteins, NF-YC3, NF-YC4, and NFYC9, physically interact with the floral-promoting NFYB2 and NF-YB3 proteins either in yeast two-hybrid or in vivo, and these three genes are required for CONSTANSmediated, photoperiod-dependent flowering in Arabidopsis thaliana.

Although many NF-Y subunits have been studied in Arabidopsis and other species, especially in regulating flowering time, the role of many NF-Y members in $T$. aestivum is currently unknown. In the present study, one of the NF-YB family genes in wheat, namely $T a N F-Y B 3$, was identified and its expression pattern was analyzed. It was found to respond to stress, light, and vernalization.

\section{Materials and methods}

\section{Plant material and growth conditions}

Bread wheat (T. aestivum, $2 \mathrm{n} \times 6 \mathrm{x}=42$, genome constitution AABBDD) cv. 'Yannong 15', a winter habit cultivar, was grown in a controlled growth chamber at $23^{\circ} \mathrm{C}$ under long days (LD, $16 \mathrm{~h}$ light $/ 8 \mathrm{~h}$ dark) or short days
(SD, $10 \mathrm{~h}$ light/14 h dark) conditions. Leaves of 21-dayold plants grown under LD were used for the cDNA cloning for TaNF-YB3.

In the expression analysis experiment, roots of 7-dayold seedlings germinated in $10 \mathrm{~cm}$ petri plates with water under LD were harvested after $6 \mathrm{~h}$ of light for total RNA extraction. Various tissues, including stems, flag leaves, the third leaves numbered from the top of plants, young spikes $1.5-2.0 \mathrm{~cm}$ in length, stamens, pistils, and young embryos of heading wheat plants grown under LD condition were also harvested after $6 \mathrm{~h}$ of light for total RNA extraction.

The first leaves from the base of plants, grown under both LD and SD conditions, were sampled 1, 2, 3, 4, 5, $6,7,8,9$, and $10 \mathrm{wk}$ after $6 \mathrm{~h}$ of light. In addition, wheat plants grown under LD for $21 \mathrm{~d}$ were shifted to SD for $6 \mathrm{wk}$, followed by $1 \mathrm{wk}$ under LD (LD-SD). Samples from plants were similarly taken $1,2,3,4,5,6$, and 7 wk after the shift to SD treatment at identical time points. For the vernalization treatment, 21 -day-old plants grown at $23^{\circ} \mathrm{C}$ under $\mathrm{LD}$ were either transferred to $\mathrm{SD}$ at $23^{\circ} \mathrm{C}$ as the control condition, or transferred to $\mathrm{SD}$ at $4^{\circ} \mathrm{C}$ for vernalization. Six weeks later, the two treated plants were transferred to a chamber at $23^{\circ} \mathrm{C}$ under LD for $1 \mathrm{wk}$.

For the stress treatment, 14-day-old wheat plants in pots under $\mathrm{LD}$ at $23^{\circ} \mathrm{C}$ were treated as follows: for salt, mannitol, and ABA, the leaves of plants grown after $10 \mathrm{~h}$ light were obtained by dipping the plants in $300 \mathrm{mM}$ $\mathrm{NaCl}, 300 \mathrm{mM}$ mannitol, and $100 \mu \mathrm{M}$ ABA solution, respectively, for $16 \mathrm{~h}$; for heat shock, they were exposed at $40^{\circ} \mathrm{C}$ for $1 \mathrm{~h}$; wounding stress was induced by cutting seedlings into $1.5 \mathrm{~cm}$ segments and placing them in water for $14 \mathrm{~h}$; the drought-stressed plants were treated without water for $1 \mathrm{wk}$; for cold treatment, the leaves were taken 2 , $4,12,24$, and $48 \mathrm{~h}$ after the plants were transferred to $4^{\circ} \mathrm{C}$ under LD condition.

For the light regulation experiment, 12-day-old plants grown at $23^{\circ} \mathrm{C}$ under $\mathrm{LD}$ condition were used for treatment. For the first designed experiment, the plants were transferred to darkness after being kept for $48 \mathrm{~h}$ in light, and their leaves were harvested $1,2,3,4$, and $5 \mathrm{~h}$ after shifting to darkness. The plants were moved to the light after being kept in the dark for $48 \mathrm{~h}$, and were then taken 1, 2, 3, 4 , and $5 \mathrm{~h}$ after shifting to light. For the diurnal expression study, leaves of 12-day-old plants grown under LD or SD conditions at $23^{\circ} \mathrm{C}$ were harvested every $2 \mathrm{~h}$ for analysis.

\section{Total RNA preparation and $c D N A$ synthesis}

Total RNA was separately extracted from all samples using the plant RNA TRIzol reagent (Invitrogen) following the manufacturer's directions, followed by incubation with Rnase-free Dnase I (TaKaRa, Dalian, China) at $37^{\circ} \mathrm{C}$ for $30 \mathrm{~min}$. The first strand complementary DNA (cDNA) was synthesized using an oligo (dT18) primer from total RNA using BioTeke super RT Kit (Bioteke Corporation, China) following the manufacturer's instructions. 
256

Cloning, Chromosome Localization, and Sequence Analysis of TaNF-YB3

The wheat TaNF-YB3 gene was amplified with PCR primers designed under GenBank accession no. BT009265: NYB3-1 (5'-AGATTGAATTTTCGTACAAGTGTCC-3') and NYB3-2 (5'-TAAGCTACAAGTCACGGCTTCATC-3').

PCR amplification was performed with high-fidelity DNA polymerase ( $P f u$ DNA polymerase, Promega), followed by the addition of rTaqase (TaKaRa, Dalian, China). The PCR program was performed as follows: $94^{\circ} \mathrm{C}$ for $5 \mathrm{~min}$, followed by 35 cycles of amplification $\left(94^{\circ} \mathrm{C}\right.$ for $1 \mathrm{~min}$, $60^{\circ} \mathrm{C}$ for $40 \mathrm{~s}$, and $72^{\circ} \mathrm{C}$ for $2 \mathrm{~min}$ ). The amplified product was cloned into the pMD18-T vector ( $\mathrm{TaKaRa}$ ) followed by sequencing. Subsequent BLAST results confirmed that the fragment was the TaNF-YB3 gene, whose sequence is 809 bp long, with 5'UTR, ORF, and 3'UTR.

Genomic DNA of the wheat cultivar Chinese spring ditelocentric series, kindly provided by professor Lingrang Kong (College of Agronomy, Shandong Agricultural University, Taian, China), was used for the chromosome localization of the TaNF-YB3 gene. From the diluted genomic stocks, $4 \mu \mathrm{L}$ (30 ng) was used as a template in $25 \mathrm{~mL}$. Two primers(YB3-a,5'-ATGCCGGACTCGGACAACG-3' and YB3-b, 5'-CCCCTCTTTCCGTCCGAAC-3') based on the ORF sequence were designed for PCR amplification. The PCR thermal cycling parameters were $95^{\circ} \mathrm{C}$ for $5 \mathrm{~min}$ followed by 38 cycles at $95^{\circ} \mathrm{C}$ for $30 \mathrm{~s}, 59^{\circ} \mathrm{C}$ for $30 \mathrm{~s}$, and $72^{\circ} \mathrm{C}$ for $1 \mathrm{~min}$.

All sequences were aligned using DNAMAN 5.2.2. Phylogenetic analysis was conducted using the neighborjoiningmethod(Thompsonetal., 1997) usingCLUSTALX version 1.83 with the amino acid sequences of the conserved domain. The accession numbers of the analyzed genes are as follows: A. thaliana AtNF-YB1 (NM_179974), AtNF-YB2 (NM_124138), AtNF-YB3 (NM_117534), AtNF-YB4 (NM_100774), AtNF-YB5 (NM_130348), AtNF-YB6 (NM_124141), AtNF-YB7 (NM_126937), AtNFYB8 (AK317223), AtNF-YB9 (NM_102046), AtNF-YB10 (NM_115194), AtNF-YB11 (NM_128307), AtNF-YB12 (NM_120902), and AtNF-YB13 (NM_180730); Oryza sativa OsHĀP3A (AB095438), OsHAP3B (AB095439), OsHAP3C (AB095440), OsHAP3D(AB288035),OsHAP3E(AB288036), OsHAP3F (AB288037), OsHAP3G (AB288038), OsHAP3H (AB288039), OsHAP3I(AB288040), OsHAP3J(BR000375.1), and OsHAP3K (AJ300218); Saccharomyces cerevisiae ScHAP3 (M20138); and Homo sapiens HsNF-YB (L06145).

\section{Real-Time PCR Analysis}

Quantitative real-time PCR was performed with a CFX 96TM Real-Time System (Bio-Rad) using Real Master Mix (SYBR Green) (TIANGEN, China) for the quantification of TaNF-YB3 transcript levels in different tissues and developmental stages, and under various stress treatments, light response, and diurnal rhythmic expression. The PCR reaction (total volume $10 \mu \mathrm{L}$ ) consisted of $1 \times$ master mix, $1 \mu \mathrm{M}$ of each primer, and $1 \mu \mathrm{L}$ cDNA
(20 ng) as the template. The cycling conditions were $1 \mathrm{~min}$ at $95^{\circ} \mathrm{C}, 40$ cycles of $10 \mathrm{~s}$ at $95^{\circ} \mathrm{C}, 10 \mathrm{~s}$ at $60^{\circ} \mathrm{C}$, and $15 \mathrm{~s}$ at $68^{\circ} \mathrm{C}$, followed by a melting curve program $\left(65-95^{\circ} \mathrm{C}\right.$, with a $5 \mathrm{~s}$ hold at each temperature). Fluorescence data were acquired at the $68^{\circ} \mathrm{C}$ step and during the melting curve program. Four replicate reactions for each cDNA primer combination were performed for each sample in the same run. For real-time PCR, a primer pair (5'-TGAGGCAGACAGATCGAGGAG-3' and '5'ACGTTGGCGATGGGCAGGAAG-3', 120 bp) was designed to amplify TaNF-YB3. Previously published gene primers 5'-CCAGTACCTACACAGCTTCCA-3' and 5'-GCCTGCTTCTTCTCCTTGT-3' of TaHdl, 5'-GCACCACTTGTAGGGGCAGA-3' and 5'TTGATCCTTGGCCGTGCTT-3' of WCOI (Shimada et al., 2009), 5'-ATCACCTTCGCTGCTCTCTC-3' and 5'-CCCACATCGTGCCATTTTAC-3' of TaZC$C T 1$, and $5^{\prime}$-CCACCATCGTGCCATTCT- ${ }^{\prime}$ and 5'CCCACCATCATCTCTGTATCAA-3' of TaZCCT2 (Distelfeld et al., 2009) were synthesized for real-time PCR in the diurnal rhythmic expression; these primers can amplify all three homoeologs located on $A, B$, and D wheat genomes. Primers 5'-ACCTTCAGTTGCCCAGCAAT3 'and 5'-CAGAGTCGAGCACAATACCAGTTG-3' of Actin were used as an external control (Yan et al., 2004). The $2^{-\Delta \Delta \mathrm{Ct}}$ method (Livak and Schmittgen, 2001) was used to normalize and calibrate the transcript values relative to the endogenous control.

\section{Results}

Cloning and sequencing of TaNF-YB3 from $6 x$ wheat

The full-length cDNA sequence of TaNF-YB3 (Genbank accession No. JF830784) was obtained using RTPCR with special primers based on the NCBI data. It was $809 \mathrm{bp}$ in length and contained an open reading frame (ORF) of 597 bp encoding 199 amino acids. There was a $5^{\prime}$ untranslated region of $123 \mathrm{bp}$ upstream from the start codon, and the coding region was followed by a $3^{\prime}$ untranslated region that was 86 bp long downstream from the stop codon. Using the genomic DNA of ditelocentric wheat lines (kindly provided by Professor Lingrang Kong), the ORF sequence was mapped on chromosome 3D (Fig. 1).

The deduced TaNF-YB3 protein had a theoretical $p \mathrm{I}$ at 6.22 and a predicted mol. wt of $23.3 \mathrm{kDa}$, obtained using the software of Computer $\mathrm{pI} / \mathrm{Mw}$ Tool at http:// www.wxpasy.org/. In contrast to yeast and animals, plants have multiple genes for the NF-YB subunit. The deduced TaNF-YB3 of the 12 OsHAP3 proteins in rice and the 13 AtNF-YB proteins in Arabidopsis were compared with that of yeast and human. Results show that TaNF-YB3 showed high identity of $85 \%$ and $66 \%$ to OsHAP3I and AtNFYB3, respectively, and presented low identity of 50\% and $47 \%$ to yeast and human, respectively. The TaNF-YB3 also had a conserved domain in its centre, similar to the NF-YB 

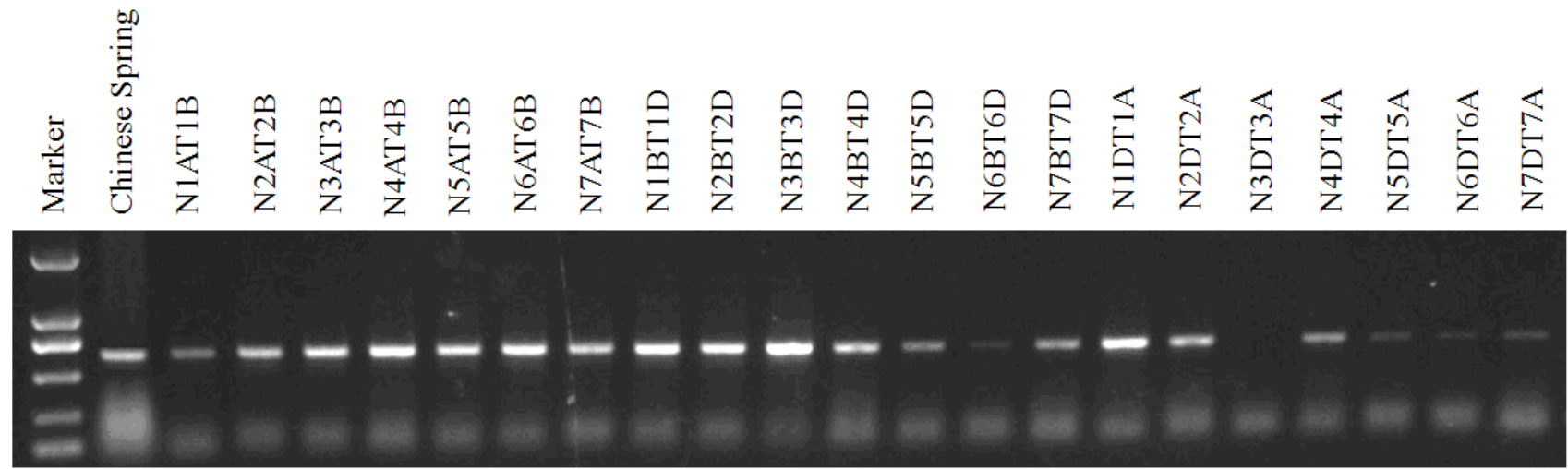

Fig. 1. PCR analysis for the determination of the chromosomal location of TaNF-YB3. The chromosomal locations ofTaNF-YB3 gene was determined by PCR in combination with genomic DNA of Chinese Spring (CS) nulli-tetrasomics lines

subunits in other species. This core region is 95 amino acids in length (Fig. 2a). Amino acids 5-36 in the conserved core region of TaNF-YB3 may well be required for DNA binding in the heterotrimer complex NF-YA/NF-YB/NFYC, residues 9-50 and 55-89 may be involved in heterodimer formation, and a short linker region (39-47 aa) could interact with the TaNF-YA subunit (Fig. 2a).
The NF-YB proteins have been divided into two classes in Arabidopsis, the LEC1-like and the non-LEC1-like (Lee et al., 2003). A phylogenetic analysis based on the core sequence showed that the NF-YB proteins were located in several different clades. Among them, TaNF-YB3 belonged to a non-LEC1-like clade, which also includes OsHAP3F and OsHAP3I (Fig. 2b), both of which showed similarities even in the non-conserved regions (Fig. 2a).

(a)

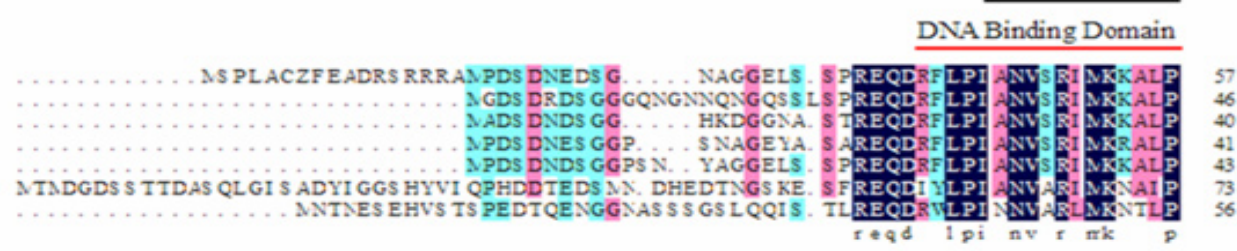

NF-YC Interaction

$\frac{\text { NF-YC Interaction }}{\text { NF-YA Interaction }}$

T2IF-YB3

ALAF.YB2

ALTF.YB3

OnHAP3I

HaNF.YB

HaNF.YB
SchLAP3

Consenves

T2YF:YB3

AtTF.IB:

ALTF-YB3

OAHAP3F
OHHAP 31
HATF

HANF-IB

Collap3

TAF.YB3

AEF.YB2

ALFF.YB3

OAHAP31

OALAP31

SAHAP3

Consenses

TIF.YB3

AETF.YB2

ALTF-YB

OAHAP3:

OAHAP31

HaNF-YB

SCHAP3

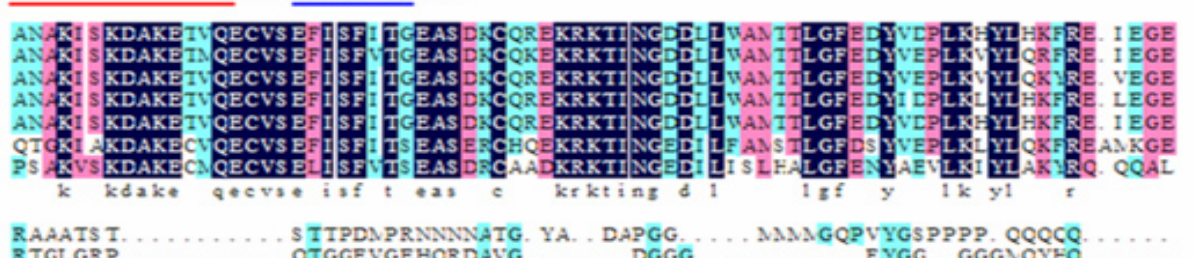

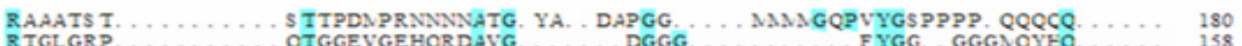

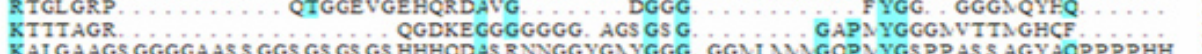

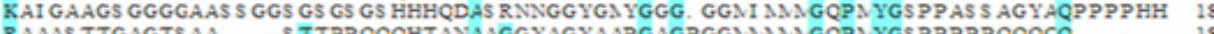

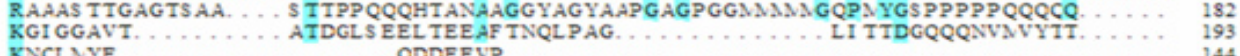

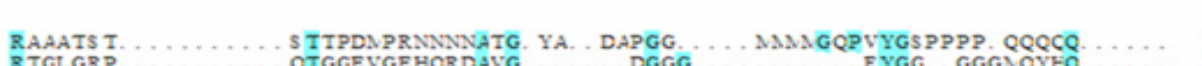

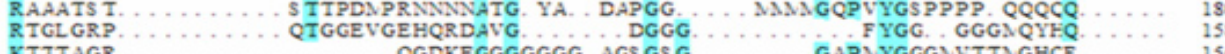

KTITAGR. RAAAS TTGAGTS A.A. ... S TTPPQQQHTANAAGGYAGYAAPGAGPGGMMAMGQPMYGSPPPPPQQQCQ ...... 18

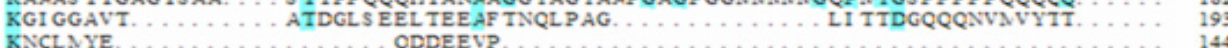

HQEQI ANGGR.AGF PYHGGGGGCGGS \$\$ S GFGRKEG.

HFQFLHQQNHAYGATGGGS DSG. GGAAS GRTRT.

S FHFS.

HHFQNVAGGKGAYG. HGGGGGCG. PS PS S GYGRQDRL

QHFHAAMGGRGGF GHHPGGGGGG. S\$SS S GEGRQNRG

SYCQI S GVQQI CFS

Fig. 2. NF-YB genes in wheat, Arabidopsis, rice, yeast and human. (a) An alignment of deduced amino acid sequences of NF-YB family proteins. Alignment was performed with DNAMAN 5.5.2. Fully, strongly and weakly conserved amino acids are shaded in black, pink, blue, respectively. Red box indicates conserved core regions. Within the conserved core regions: black lines under the alignments indicate regions involved in contacting DNA, red lines under the alignments indicate regions involved in hetero dimerisation and blue lines under the alignments indicate NF-YA interaction regions 
258 (b)

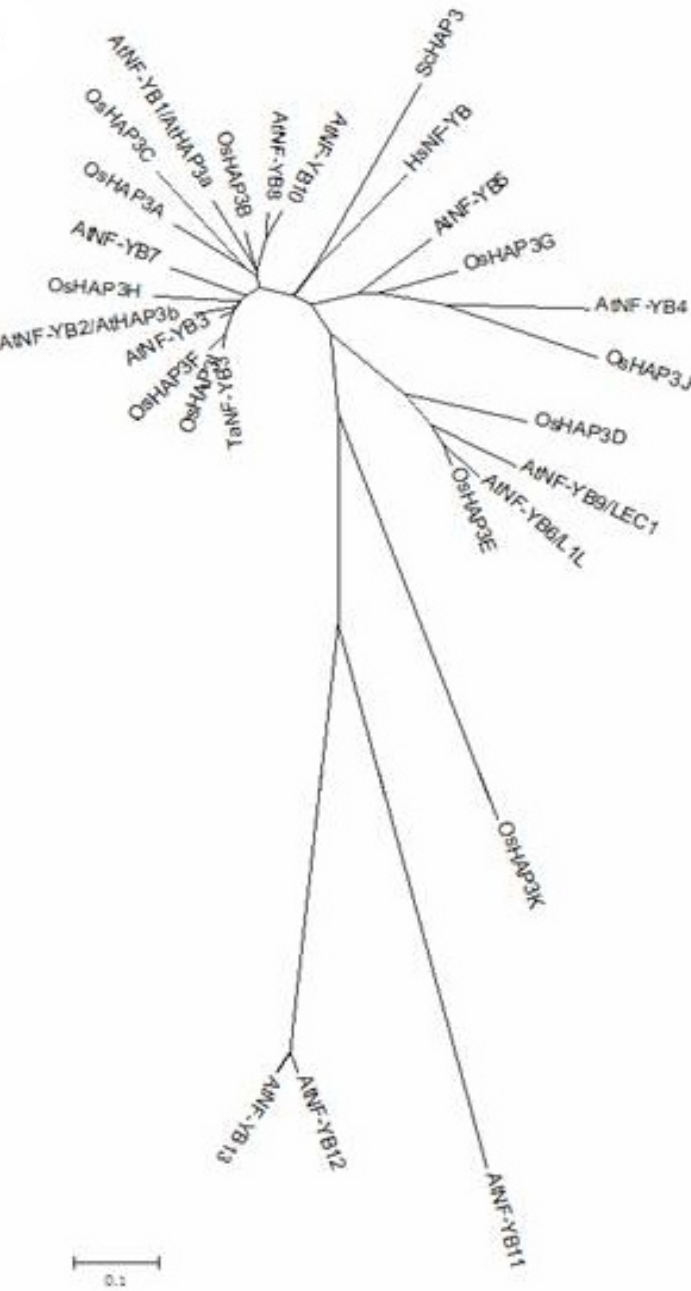

Fig. 2. NF-YB genes in wheat, Arabidopsis, rice, yeast and human. (b) A phylogenetic tree of the NF-YB proteins of wheat, rice, Arabidopsis, yeast and human. Sc, Saccharomyces cerevisiae; Hs, Homo sapiens HAP3 protein

\section{TaNF-YB3 gene expression in wheat}

RT-PCR analysis using RNA isolated from various winter wheat organs was conducted to examine the TaNF$Y B 3$ gene expression patterns. The data indicated that TaNF-YB3 transcripts accumulated mostly in the leaves, less in the stems, and was barely detectable in the roots (Fig. 3a). In addition, its expression was weakly detected in young spikes, pistils, and young embryos (Fig. 3a). However, TaNF-YB3, which belongs to the no-L1L clade, was also strongly expressed in the stamen, as high as that in the stem (Fig. 3a).

TaNF-YB3 expression was also analyzed in the vegetative stage by extracting total RNA from winter wheat leaves. Under the long day (LD) condition, the level of TaNF-YB3 transcripts gradually reached the highest level in the eighth week (Fig. 3b), whereas it had no obvious expression changes under the short day (SD) condition, and accumulated to a lesser level compared with the LD. This result suggests that the TaNF-YB3 expression pattern in winter wheat most likely depends on the LD.
Furthermore, a day-length transferred treatment experiment was performed. First, 21-day-old wheat grown under $\mathrm{LD}$ was transferred to $\mathrm{SD}$ for $6 \mathrm{wk}$, and then removed to the LD for $1 \mathrm{wk}$ (LD-SD). The TaNF-YB3 expression pattern was examined in the LD-SD experiment (Fig. 3b). TaNF-YB3 expression was extraordinarily higher at the first week after the transfer to SD, compared with that under LD. Subsequently, the expression gradually decreased to the level under LD on the 10th week. This reveals that photoperiod translation had a great effect on TaNF-YB3.

Winter wheat needs to be extendedly exposed to low temperatures to flower (vernalization). Here, vernalization treatment was conducted by simulating natural conditions to investigate the TaNF-YB3 expression pattern. TaNF-YB3 transcript accumulation was found to be distinctly downregulated by vernalization exposure (Fig. 3c). It was quickly decreased in the first week, and then slightly increased; however, it still exhibited lower levels than the control. In addition, TaNF-YB3 accumulated to a higher level than the control $1 \mathrm{wk}$ after the plant was taken out of vernalization. This observation suggests that vernalization also had a great effect on TaNF-YB3.

\section{TaNF-YB 3 is regulated by various stresses}

The effect of different types of stress on TaNF-YB3 expression was investigated because many NF-Y family members play an important role in response to stresses, such as osmotic stress, ABA, and drought (Li et al., 2008; Nelson et al., 2007; Warpeha et al., 2007). The leaves of winter wheat exposed to heat shock, wounding, drought, salt, mannitol, ABA stress, and cold responsiveness for various durations were harvested for total RNA extraction (see the Materials and Methods section). The quantitative real-time RT-PCR analysis revealed that TaNF-YB3 transcripts were mostly repressed by wounding and drought treatments (Fig. 4a), and was induced by salt $(300 \mathrm{mM}$ $\mathrm{NaCl}$ ), $300 \mathrm{mM}$ mannitol, and $100 \mu \mathrm{M} \mathrm{ABA}$ after $16 \mathrm{~h}$ of treatment. Furthermore, this gene was significantly upregulated under mannitol conditions by over a 2 -fold level. However, TaNF-YB3 mRNA levels showed no change when subjected to heat shock stress at $40^{\circ} \mathrm{C}$ for $3 \mathrm{~h}$ (Fig. 4a). These results indicate that TaNF-YB3 may be involved in the response to some stresses.

As shown in Fig. 4b, TaNF-YB3 expression obviously decreased under cold treatment. It reached the lowest level at $4 \mathrm{~h}$ and then slightly increased; however, it still exhibited a lower level than the control by at least 2-8 fold. These data further confirmed the inducible expression of TaNF$Y B 3$ under stress conditions.

\section{TaNF-YB 3 regulation by light}

Two different experiments were designed to test the response of TaNF-YB3 transcript levels to light. In the first experiment, 12-day-old plants of $T$. aestivum cv 'Yannong $15^{\prime}$ (winter growth habit) grown at $23^{\circ} \mathrm{C}$ under $\mathrm{LD}$ condition were transferred to darkness after being kept for $48 \mathrm{~h}$ of light. As shown in Fig. 5a, the TaNF-YB3 expression 
(a)

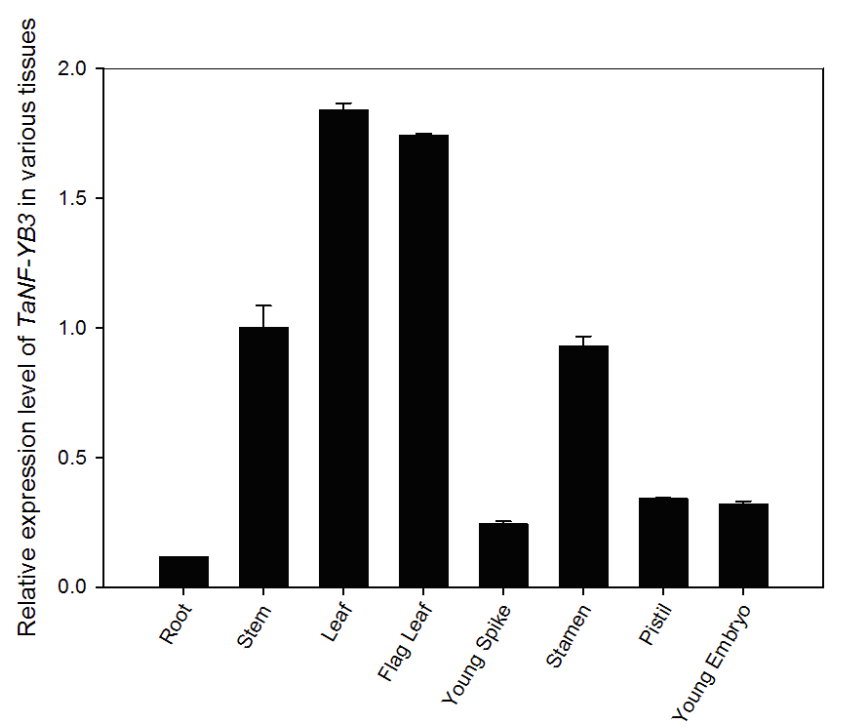

(c) (b)

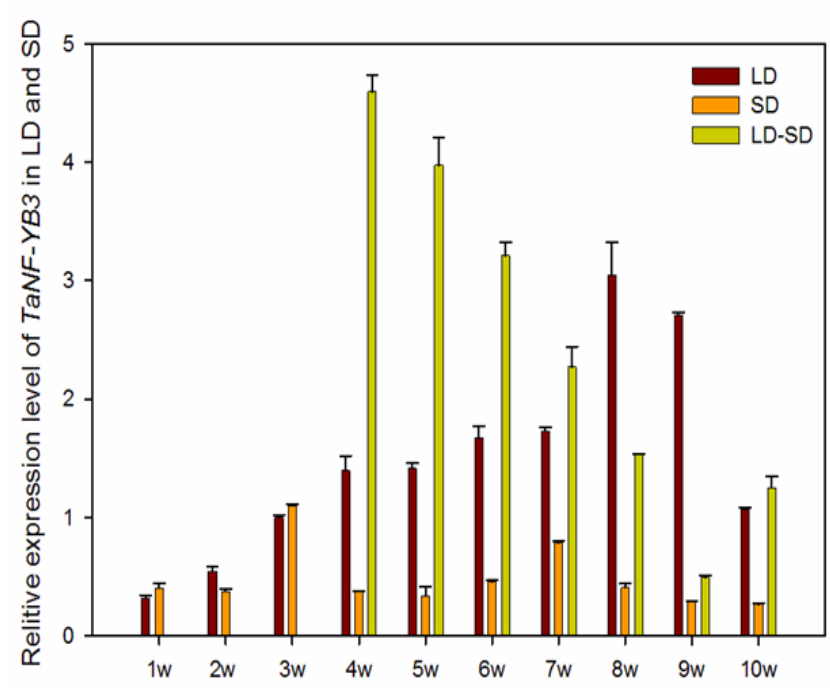

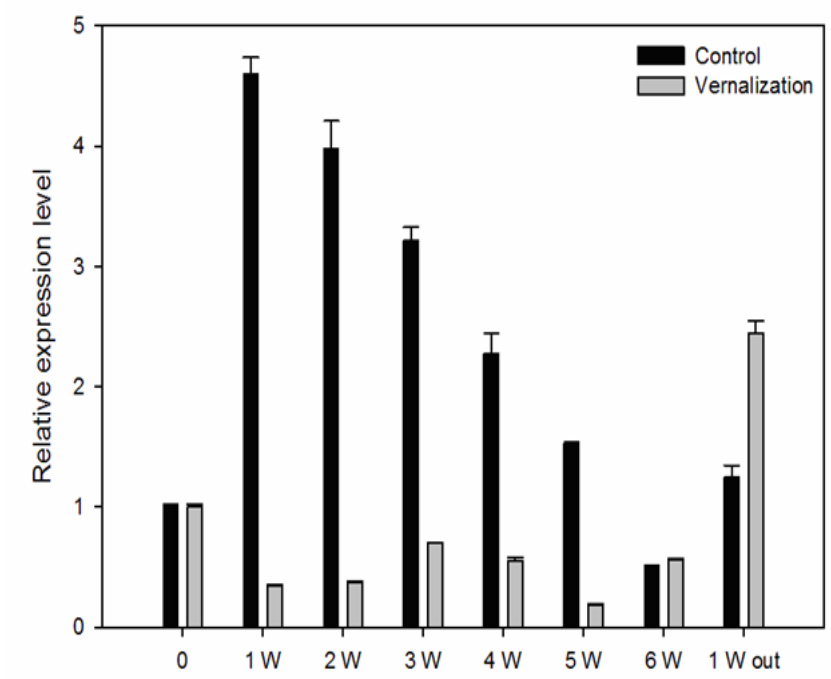

Fig. 3. Expression of TaNF-YB3 gene in wheat. (a) RT-PCR analysis of TaNF-YB3 transcripts in various tissues of wheat plants. (b) TaNF-YB3 expression patterns in different developmental stages of wheat plants grown under long day or short day conditions. (c) Expression pattern of TaNF-YB3 in vernalization

level was strongly downregulated and exhibited the lowest level after $2 \mathrm{~h}$ of darkness. Subsequently, the expression level slightly increased; however, it was still lower than in the light. In the second experiment, the plants were moved to the light after being kept for $48 \mathrm{~h}$ in darkness. TaNF$Y B 3$ also exhibited a higher level in light than in dark; the level gradually decreased in light until after $4 \mathrm{~h}$ (Fig. 5b).

Diurnal rhythmic expression patterns of TaNF-YB3, CO and VRN2 in wheat leaves

In Arabidopsis, some researchers have reported that CONSTANS (CO), which encodes a CCT-domain (CO, CO-like, and TOC1) protein, replaced AtHAP2 in the HAP complex to form a trimeric CO/AtHAP3/AtHAP5 complex (Wenkel et al., 2006). The wheat $C O$ and $H A P s$ (NF-Y genes) should have a similar diurnal rhythmic ex- pression pattern if the trimeric $\mathrm{CO} / \mathrm{AtHAP} 3 / \mathrm{AtHAP5}$ complex exists in wheat. Hence, two wheat $C O$ candidate genes (WCOI and TaHdl) (Shimada et al., 2009) and the wheat $V R N 2$ gene, which contains ZCCT1 and ZCCT2 at its locus and has a CCT-domain similar to that found in CO (Distelfeld et al., 2009), were chosen together with TaNF-YB3 for the investigation of their diurnal rhythmic expression patterns. Leaves at the three-leaf stage in winter wheat T. aestivum cv. 'Yannong 15' plants grown under $\mathrm{LD}$ or SD conditions were used to test the pattern.

Under LD conditions, the two $C O$ candidate gene ( $W C O 1$ and TaHdl) mRNA accumulated during the dark period, and $W C O 1$ expression peaked $2 \mathrm{~h}$ later than that of TaHd1 (Fig. 6a). In contrast, VRN2 (TaZCCT1 and TaZCCT2) mRNA accumulated during the light period with a dual peak (Fig. 6c). Both TaZCCT1 and TaZCCT2 
260

(a)

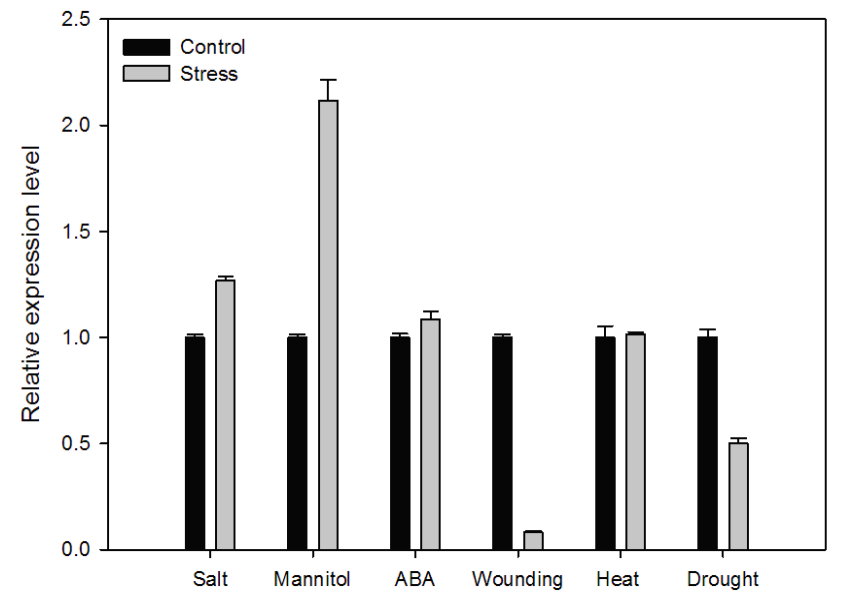

(b)

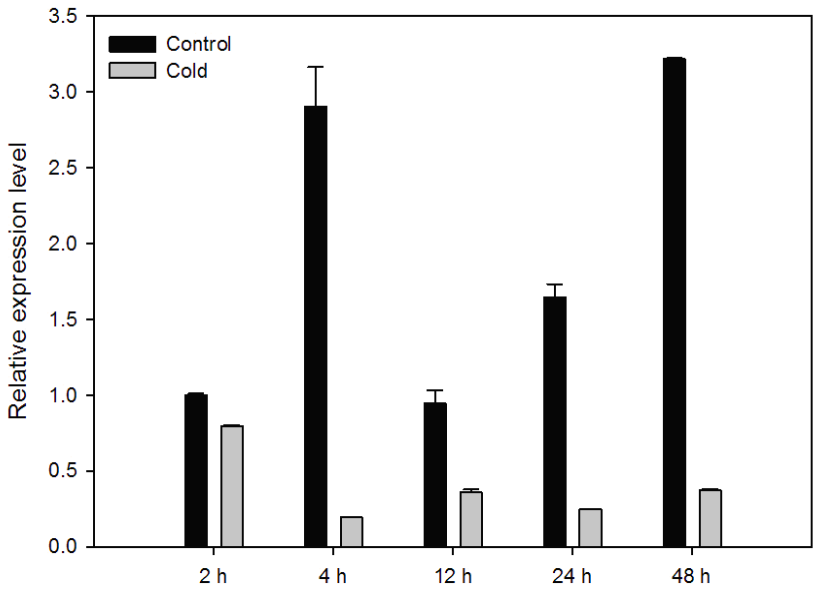

Fig. 4. Transcript levels of TaNF-YB3 in response to various stresses. (a)TaNF-YB3 expression levels of salt, mannitol, abscisic acid(ABA), wounding, heat shock and drought stresses. (b)Relative quantities of TaNF-YB3 mRNA at various time points after treated with cold at $4^{\circ} \mathrm{C}$ under $\mathrm{LD}$ condition

(a)

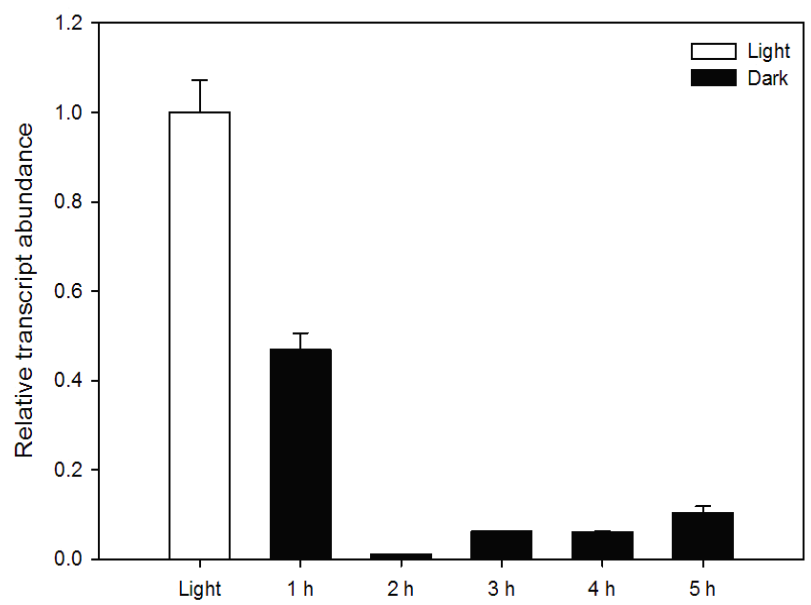

(b)

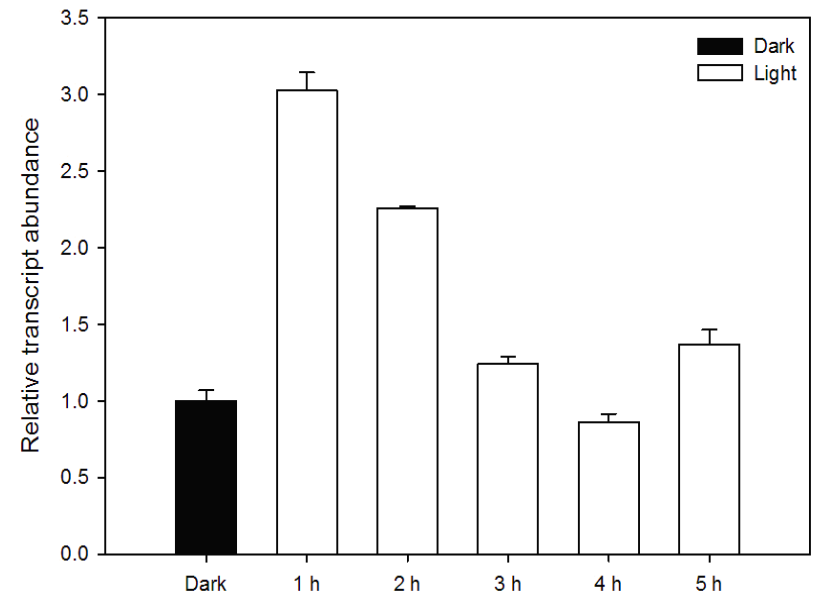

Fig. 5. Expression of TaNF-YB3 under different light conditions in winter wheat. (a) Expression of TaNF-YB3 in wheat leaves when plants were transferred from light to dark. (b) Expression of TaNF-YB3 in wheat leaves when plants were transferred from dark to light

showed a peak at the beginning of the $2 \mathrm{~h}$ light period, and TaZCCT2 showed the highest level at the end of the light period, whereas TaZCCT1 expression peaked 2 h earlier compared with TaZCCT2. TaNF-YB3 exhibited a similar pattern with VRN2, especially with TaZCCT2 (Fig. 6e). Its mRNA accumulated from the beginning of the light period, and also showed the highest level at the end.

Under SD conditions, WCOI and TaHd 1 also showed a pattern similar with each other, and their mRNA accumulated during the dark period in the same way as under LD conditions, except for a dual peak displayed in this period (Fig. 6b). Similarly, VRN2 (TaZCCT1 and TaZCCT2) mostly expressed during the light period and exhibited a dual peak expression pattern, with the higher peak late in the light period compared with that under LD conditions (Fig. 6d). Interestingly, TaNF-YB3 expression also showed a similar pattern with $V R N 2$ (TaZCCT1 and TaZCCT2) (Fig. 6f). Moreover, in both TaNF-YB3 and TaZCCT2, one peak was observed early under $4 \mathrm{~h}$ of light, and another peak with higher levels appeared at the end of $10 \mathrm{~h}$ light).

These results indicate that the wheat TaNF-YB3 was expressed in a rhythmic manner in leaves under both LD and SD conditions. It exhibited a different expression pattern from that of $C O$ ( $W C O 1$ and TaHdl), but had a similar pattern with VRN2 (TaZCCT1 and TaZCCT2), especially with TaZCCT2, either under LD or SD conditions. 
LD
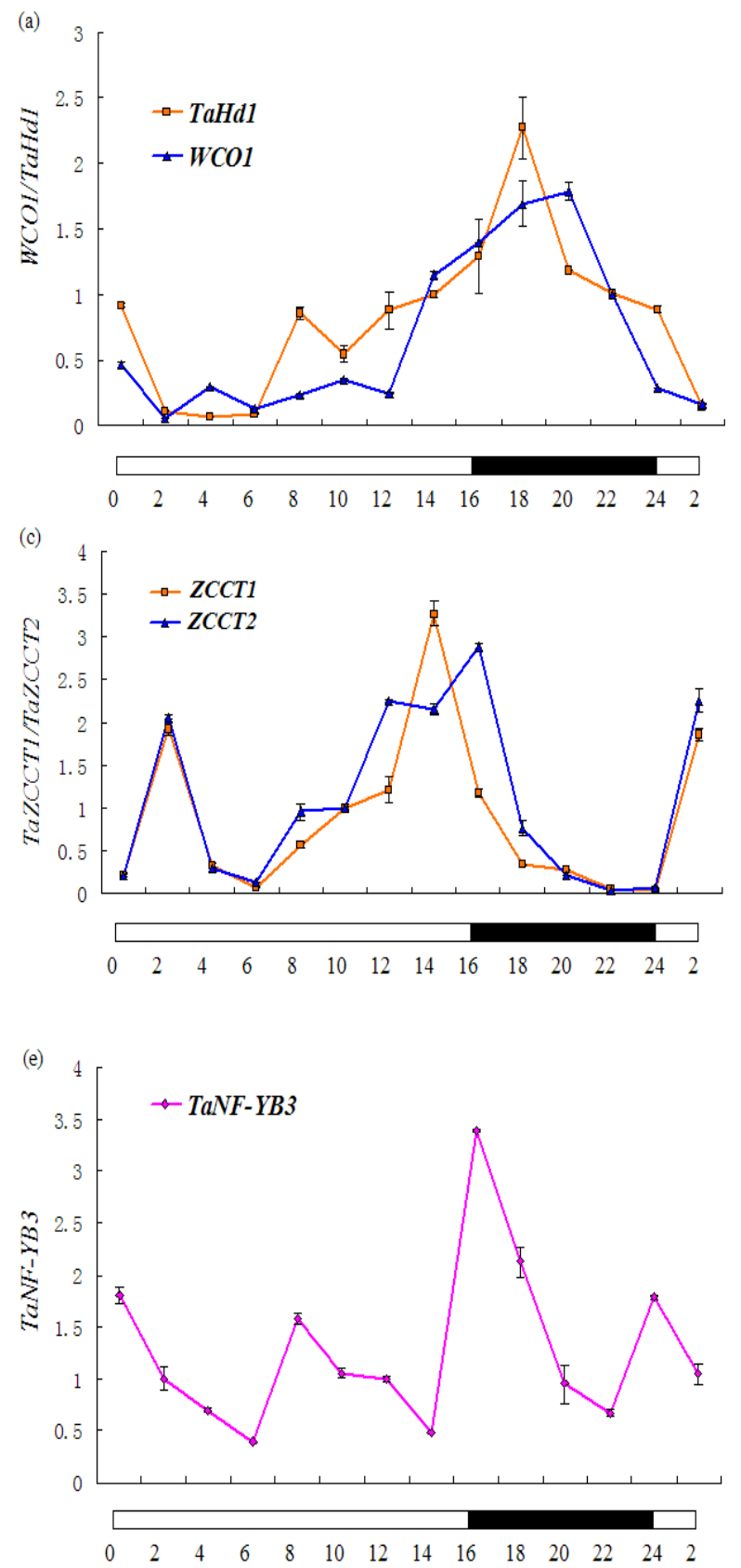

SD
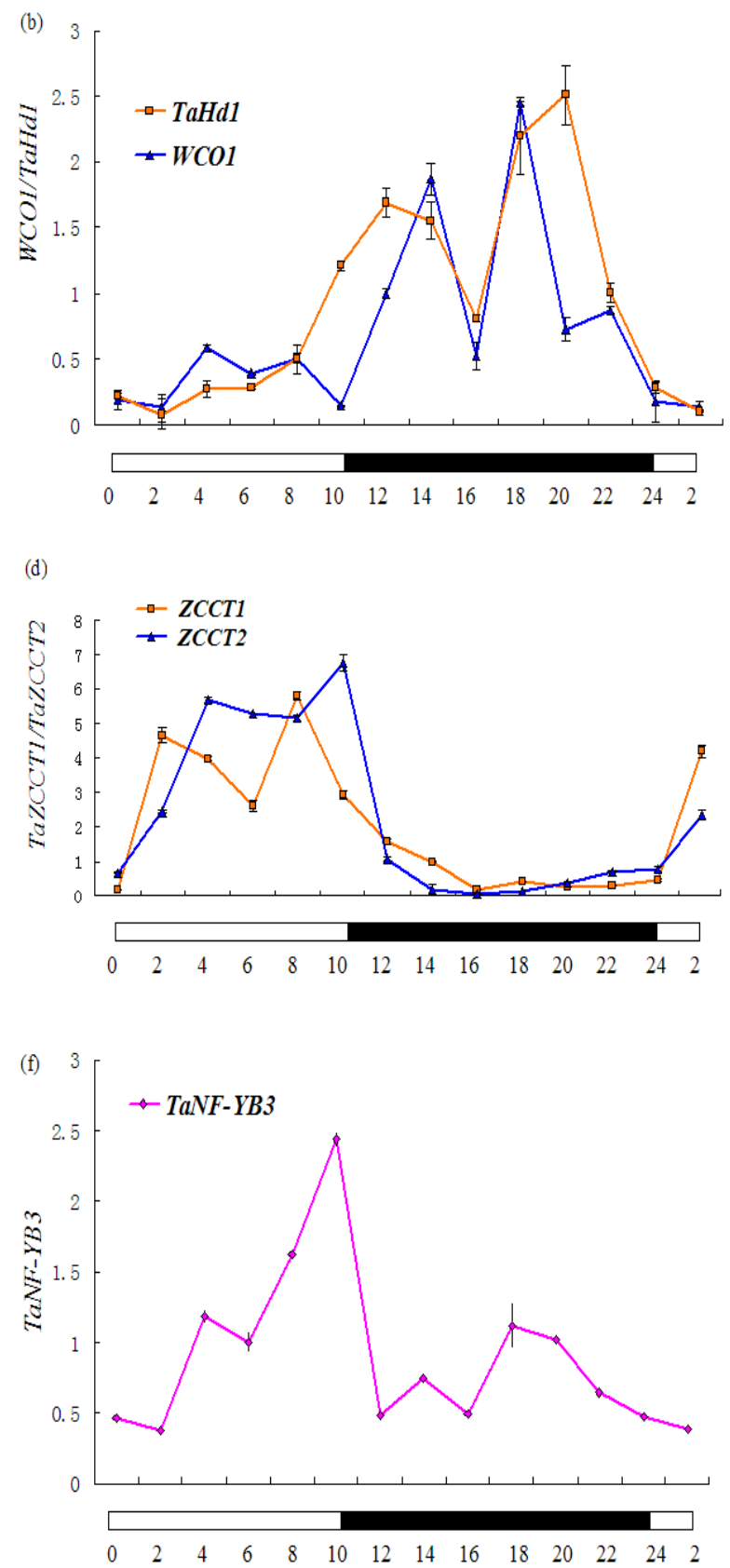

Fig. 6. Diurnal expression patterns of TaNF-YB3,CO and TaVRN2 in winter wheat plants, grown under long days (LD) and short days (SD) conditions. The expression patterns of TaNF-YB3, CO (WCO1 and TaHd1), and TaVRN2 (TaZCCT1 and TaZCCT2) were analyzed by real-time PCR using winter wheat plants at the three-leaf stage grown under LDs (16 h light/8 h dark) (a) or SDs $(10 \mathrm{~h} \mathrm{light} / 14 \mathrm{~h} \mathrm{dark})(\mathrm{b})$ at $23^{\circ} \mathrm{C}$. The Actin gene was used as an internal control for calculating the relative levels of the above genes. Each point represents the average of three replicates, and the error bars indicate the range. The white and black bars along the horizontal axes represent light and dark periods, respectively. The numbers on the horizontal axes indicate the time in hours

\section{Discussion}

A wheat NF-YB (HAP3) gene, TaNF-YB3, which was located on the chromosome 3D, was characterized. Phylogenetic analysis based on the core sequence has categorized it as belonging to the non-LEC1-like clade, which includes rice OsHAP3F and OsHAP3I (Fig. 2b). Furthermore, TaNF-YB3 exhibited a ubiquitous expression, and showed a similar expression pattern with $O s H A P 3 F$, which are expressed in young leaves, 0 -day and 8-day-after-pollination 
262

embryos, and had very weak signals in roots (Thirumurugan et al., 2008; Fig. 3a). However, OsHAP3I showed no expression in many rice organs and at the developmental stages, and seemed to be expressed in etiolated seedlings (Thirumurugan et al., 2008). Thus, expression and phylogenetic analyses of the TaNF-YB3 and other $N F-Y B$ genes revealed that the identified phylogenetic relationships were reflected by their expression profiles to some degree. Although the TaNF-YB3 gene belongs to the non-LEC1like clade, it is strongly expressed in the stamen. TaNF-YB3 was thought to be a redundancy or a form of the NF-Y trimer complex with other NF-Y members in the stamen.

The TaNF-YB3 gene was shown to be regulated by different environmental cues by examining the effects of different day lengths and vernalization treatments on $\mathrm{TaNF}$ YB3 expression in wheat. Generally, TaNF-YB3 expression gradually increased until the eighth week, and distinctly decreased after $3 \mathrm{wk}$ under SD conditions or after shifting the 21-day-old plants under LD condition to SD conditions for $6 \mathrm{wk}$ vernalization at $4^{\circ} \mathrm{C}$ (Fig. 3). During the $6 \mathrm{wk}$ of vernalization and on the 4 th to 9 th week under SD condition, TaNF-YB3 showed a similar expression pattern with a peak at the fourth or third week after the transfer. In addition, when the 21-old-day plants grown under LD conditions were transferred to SD conditions, TaNF-YB3 was extraordinarily up-regulated during the initial weeks and showed higher levels than those under LD conditions. This phenomenon indicates that day length translation had a significant effect on TaNF-YB3. However, whether day length or vernalization plays a higher effect on TaNF-YB3 is unclear. Furthermore, whether the SD condition could replace vernalization in its role in plant development, as previously reported about wheat $V R N 2$ (Dubcovsky et al., 2006), would be of great interest.

The TaNF-YB3 gene was also regulated by salt, mannitol, ABA, wounding, drought, and cold (Fig. 4). This indicates that TaNF-YB3, aside from having a role in regulating day length and vernalization responses, may integrate signals from other environmental stresses to execute its functions in winter wheat adaptability and development.

Under both LD and SD conditions, diurnal expression analysis revealed that TaNF-YB3 exhibited a similar pattern with the CCT-domain family gene, VRN2 (TaZCCT1 and TaZCCT2), especially with TaZCCT2 (Figs. 6c and 6e), but not with $C O$ (WCOI and TaHd1). This trend was supported by the observation that both TaNF-YB3 and VRN2 (TaZCCT1 and TaZCCT2) mRNA accumulated during the light period, and their expression peaked late in the light phase. However, $\mathrm{CO}$ (WCOI and TaHdI) mRNA accumulated during the dark period either under LD or SD conditions as reported in previous studies (Shimada et al., 2009; Fig. 6a and 6b). It was also based on the finding that TaNF-YB3 expressed higher in the light than in the dark (Fig. 5). In addition, Arabidopsis $\mathrm{CO}$ was reported to replace AtHAP2 in the HAP complex to form a trimeric $\mathrm{CO} / \mathrm{AtHAP} 3 / \mathrm{AtHAP} 5$ complex (Wenkel et al., 2006). Based on the results, wheat VRN2 may possibly replace TaNF-YA to form a trimeric VRN2/TaNF-YB3/ TaNF-YC complex in wheat. Further studies on VRN2 and HAP interactions, as well as on TaNF-YB3 functions, should be conducted.

\section{Acknowledgements}

The work was supported by the major research project of Shandong Province (2009ZHZX1A1401).

\section{References}

Ben-Naim O, Eshed R, Parnis A, Teper-Bamnolker P, Shalit A, Coupland G, Samach A, Lifschitz E (2006). The CCAAT binding factor can mediate interactions between CONSTANS-like proteins and DNA. Plant J 46:462-476.

Bucher P (1990). Weight matrix descriptions of four eukaryotic RNA polymerase II promoter elements derived from 502 unrelated prolaboratory. J Mol Biol 212:563-578.

Cai X, Ballif J, Endo S, Davis E, Liang M, Chen D, DeWald D, Kreps J, Zhu T, Wu Y (2007). A putative CCAAT-binding transcription factor is a regulator of flowering timing in Arabidopsis. Plant Physiol 145:98-105.

Chen NZ, Zhang XQ, Wei PC, Chen QJ, Ren F, Chen J, Wang $\mathrm{XC}$ (2007). AtHAP3b plays a crucial role in the regulation of flowering time in Arabidopsis during osmotic stress. J Bio Chem Mol Biol 40:1083-1089.

Combier JP, Frugier F, de Billy F, Boualem A, El-Yahyaoui F, Moreau S, Vernié T, Ott T, Gamas P, Crespi M, Niebel A (2006). MtHAP2-1 is a key transcriptional regulator of symbiotic nodule development regulated by microRNA169 in Medicago truncatula. Genes Dev 20:3084-3088.

Distelfeld A, Tranquilli G, Li C, Yan L, Dubcovsky J (2009). Genetic and molecular characterization of the VRN2 loci in tetraploid wheat. Plant Physiol 149:245-257.

Dorn A, Durand B, Marfing C, Le MM, Benoist C, Mathis D (1987). Conserved major histo compatibility complex class II boxes-X and-Y are transcriptional control elements and specifically bind nuclear proteins. Proc Natl Acad Sci USA 84:6249-6253.

Dubcovsky J, Loukoianov A, Fu D, Valarik M, Sanchez A, Yan $\mathrm{L}$ (2006). Effect of photoperiod on the regulation of wheat vernalization genes VRN1 and VRN2. Plant Molec Biol 60:469-480.

Edwards D, Murray JA, Smith AG (1998). Multiple genes encoding the conserved CCAAT-box transcription factor complex are expressed in Arabidopsis. Plant Physiol 117:1015-1022.

Forsburg SL, Guarente L (1989). Identification and characterization of HAP4: a third component of the CCAAT-bound HAP2 HAP3 heteromer. Genes Dev 3:1166-1178.

Gusmaroli G, Tonelli C, Mantovani R (2001). Regulation of the CCAAT-binding NF-Y subunits in Arabidopsis thaliana. Gene 264:173-185. 
Gusmaroli G, Tonelli C, Mantovani R (2002). Regulation of novel members of the Arabidopsis thaliana CCAAT-binding nuclear factor $Y$ subunits. Gene 283:41-48.

Kwong RW, Bui AQ, Lee H, Kwong LW, Fischer RL, Goldberg RB. Harada JJ (2003). Leafy cotyledon1-like defines a class of regulators essential for embryogenesis. Plant Cell 15:5-18.

Kumimoto RW, Adam L, Hymus GJ, Repetti PP, Reuber TL, Marion CM, Hempel FD, Ratcliffe OJ (2008). The nuclear factor Y subunits NF-YB2 and NF-YB3 play additive roles in the promotion of flowering by inductive long-day photoperiods in Arabidopsis. Planta 228:709-723.

Kumimoto RW, Zhang Y, Siefers N, Holt III BF (2010). NFYC3, NF-YC4 and NF-YC9 are required for CONSTANSmediated, photoperiod-dependent flowering in Arabidopsis thaliana. Plant J 63:379-391.

Lee HS, Fischer RL, Goldberg RB, Harada JJ (2003). Arabidopsis leafy cotyledon 1 represents a functionally specialized subunit of the CCAAT binding transcription factor. Proc Natl Acad Sci USA 100:2152-2156.

Li WX, Oono Y, Zhu J, He XJ, Wu JM, Iida K, Lu XY, Cui X, Jin H, Zhu JK (2008). The Arabidopsis NFYA5 transcription factor is regulated transcriptionally and posttranscriptionally to promote drought resistance. Plant Cell 20:2238-2251.

Livak KJ, Schmittgen TD (2001). Analysis of relative gene expression data using real-time quantitative PCR and the $2^{-\Delta \Delta C T}$ method. Methods 25:402-408.

Li XY, Mantovani R, Vanhuijsduijnen RH, Andre I, Benoist C Mathis D (1992). Evolutionary variation of the CCAATbinding transcription factor NF-Y. Nucleic Acids Res 20:1087-1091.

Lotan T, Ohto M, Yee KM, West MA, Lo R, Kwong RW, Yamagishi K, Fischer RL, Goldberg RB, Harada JJ (1998). Arabidopsis leafy cotyledon 1 is sufficient to induce embryo development in vegetative cells. Cell 93:1195-1205.

Maity SN, de Crombrugghe B (1998). Role of the CCAATbinding protein $\mathrm{CBF} / \mathrm{NF}-\mathrm{Y}$ in transcription. Trends Biochem Sci 23:174-178.

Mantovani R (1998). A survey of 178 NF-Y binding CCAAT boxes. Nucleic Acids Res 26:1135-1143.

Mantovani R (1999). The molecular biology of the CCAATbinding factor NF-Y. Gene 239:15-27.

McNabb DS, Tseng KA, Guarente L (1997). The Saccharomyces cerevisiae HAP5p homolog from fission yeast reveals two conserved domains that are essential for assembly of heterotetrameric CCAAT binding factor. Mol Cell Biol 17:7008-7018.

Miyoshi K, Ito Y, Serizawa A, Kurata N (2003). OsHAP3 genes regulate chloroplast biogenesis in rice. Plant J 36:532-540.
Nelson DE, Repetti PP, Adams TR, Creelman RA, Wu J, Warner DC, Anstrom DC, Bensen RJ, Castiglioni PP, Donnarummo MG, Hinchey BS, Kumimoto RW, Maszle DR, Canales RD, Krolikowski KA, Dotson SB, Gutterson N, Ratcliffe OJ, Heard JE (2007). Plant nuclear factor Y (NF-Y) B subunits confer drought tolerance and lead to improved corn yields on water-limited acres. Proc Natl Acad Sci USA 104:16450-16455.

Romier C, Cocchiarella F, Mantovani R, Moras D (2003). The NF-YB/NF-YC structure gives insight into DNA binding and transcription regulation by CCAAT factor NF-Y.J Biol Chem 278:1336-1345.

Shimada S, Ogawa T, Kitagawa S, Suzuki T, Ikari C, Shitsukawa N, Abe T, Kawahigashi H, Kikuchi R, Handa H, Murai K (2009). A genetic network of flowering-time genes in wheat leaves, in which an APETALA1/FRUITFULL-like gene, $V R N 1$, is upstream of FLOWERING LOCUS T. The Plant J 58:668-681.

Stephenson TJ, McIntyre CL, Collet C, Xue GP (2007). Genome-wide identification and expression analysis of the NF-Y family of transcription factors in Triticum aestivum. Plant Mol Biol 65:77-92.

Thirumurugan T, Ito Y, Kubo T, Serizawa A, Kurata N (2008). Identification, characterization and interaction of HAP family genes in rice. Mol Genet Genom 279:279-289.

Thompson JD, Gibson TJ, Plewniak F, Jeanmougin F, Higgins DG (1997). The CLUSTAL_X windows interface: flexible strategies for multiple sequence alignment aided by quality analysis tools. Nuc Acids Res 25:4876-4882.

Warpeha KM, Upadhyay S, Yeh J, Adamiak J, Hawkins SI, Lapik YR, Anderson MB, Kaufman LS (2007). The GCR1, GPA1, PRN1, NF-Y signal chain mediates both blue light and abscisic acid responses in Arabidopsis. Plant Physiol 143:1590-1600.

Wenkel S, Turcka F, Singer K, Gissot L, Le Gourrierec J, Samach A, Coupland G (2006). CONSTANS and the CCAAT box binding complex share a functionally important domain and interact to regulate flowering of Arabidopsis. Plant Cell 18:2971-2984.

Yan L, Loukoianov A, Blechl A, Tranquilli G, Ramakrishna W, San MP, Bennetzen JL, Echenique V, Dubcovsky J (2004). The wheat VRN2 gene is a flowering repressor downregulated by vernalization. Science 303:1640-1644.

Yazawa K, Takahata K, Kamada H (2004). Isolation of the gene encoding carrot leafy cotyledon 1 and expression analysis during somatic and zygotic embryogenesis. Plant Physiol Bio Chem 42:215-223. 